\title{
Computational Study of Resonantly Ionizing Rubidium Vapor by Nanosecond Laser Pulses
}

\author{
Mohamed Abd El-Hameid Mahmoud ${ }^{1}$, Yosr Ez El Din Gamal ${ }^{2,3}$ \\ ${ }^{1}$ Department of Physics, Faculty of Science, Sohag University, Sohag, Egypt \\ ${ }^{2}$ Department of Physics, Girls' Faculty of Science, King Abdul-Aziz University, Jeddah, KSA \\ ${ }^{3}$ National Institute of Laser Enhanced Science, Cairo University, Giza, Egypt \\ Email: hameideg@yahoo.com
}

Received June 30, 2012; revised July 31, 2012; accepted August 8, 2012

\begin{abstract}
An investigation of the resonant interaction of the rubidium atoms with an intensity $\left(10 \mathrm{~kW} \cdot \mathrm{cm}^{-2} \leq \mathrm{I} \leq 2 \mathrm{MW} \cdot \mathrm{cm}^{-2}\right)$ and a wavelength close to that of the $\mathrm{D}_{1}$ and $\mathrm{D}_{2}$ transitions of the rubidium atom $\left(5 \mathrm{~S}_{1 / 2} \rightarrow 5 \mathrm{P}_{3 / 2}\right.$ or $5 \mathrm{~S}_{1 / 2} \rightarrow 5 \mathrm{P}_{1 / 2}, \lambda_{\mathrm{D} 1}=780$ $\left.\mathrm{nm}, \lambda_{\mathrm{D} 2}=795 \mathrm{~nm}\right)$, which has passes through rubidium vapor with density $\left(10^{11}-10^{14} \mathrm{~cm}^{-3}\right)$ been studied theoretically. The system of equations describing the processes of Collisional ionization and multiphoton ionization of rubidium vapour resonantly excited with nanosecond pulsed laser is solved. The dependence of the ion density on the laser intensity and the atomic density of rubidium are considered. The result of calculations revealed that, both quadratic ion density dependence on laser intensity and linear behaviour of the ion density versus rubidium density for $5 \mathrm{~S}_{1 / 2} \rightarrow 5 \mathrm{P}_{3 / 2}$ transition is due to photoioization process. In contrast, for $5 \mathrm{~S}_{1 / 2} \rightarrow 5 \mathrm{P}_{1 / 2}$ transition, the ion density dependence is nonlinear and indicates that the collisional processes play the major contribution in the total ionization. Also, the obtained results showed reasonable agreement with the experimentally measured values of the ion density dependence given by Bakhramov et al. In addition, the analysis of mutual competition between the different ionization processes considered for the ion yield as a function of both laser intensity and atoms density are also presented this work.
\end{abstract}

Keywords: Laser; Plasma; Collisional Ionization; Electron Energy Distribution Function; Photoionization; Rubidium

\section{Introduction}

Laser-induced collisional ionization has been extensively studied ever since high-intensity pulsed lasers have been developed. In applied science, the laser-plasma mechanism is important in various studies like laser fusion, energy conversion, optical switching [1], and others, while in pure science, laser breakdown is involved in studies like ion spectroscopy [2].

Therefore, laser induced collisional ionization of $\mathrm{D}_{1,2^{-}}$ excited atoms represents considerable interest from the point of view of both the physical mechanisms and its efficiency as well as applications [3].

In low-temperature plasma physics, collisional ionization processes are traditionally regarded as providing an effective channel for the production of molecular ions. This refers in the first instance to studies involving inertgas and alkali-metal atoms in the lowest of all the possible (resonance) excited states because of their extensive applications in science and technology [3]. Two possibilities are then found to arise. They are:

1) $\sum E^{*}>U_{i}$

2) $\sum E^{*}<U_{i}$ where $\sum E^{*}$ is the total excitation energy, $U_{i}$ is the ionization potential of an individual atom. Case (1) includes the ionization of the hydrogen atoms, inert gases, the halogens, nitrogen, and oxygen, whereas Case (2) includes the ionization of alkali-metals, rare-earth elements, uranium, etc.

Since the initial studies on $\mathrm{Rb}[4,5]$ many investigations have been made to understand the ionization processes in laser-excited $\mathrm{Rb}$ vapour [6,7]. Several of these processes, associative ionization, Hornbeck-Molnar ionization and Penning ionization following energy pooling collisions, have already been identified as important factors producing primary seed electrons in $\mathrm{Rb}$ vapour irradiated by laser light tuned the frequency of the doublet resonance $5 \mathrm{~S}-5 \mathrm{P}$ over a wide range of atomic densities $\left(10^{11}-2 \times 10^{13} \mathrm{~cm}^{-3}\right)$.

The theoretical approach of laser ionization by resonant light for long laser pulses (hundreds of nanoseconds) was given by Measures et al. [8] and Bobin and Zaibi [9]. While, the model of few nanoseconds exciting pulses is presented by Stacewicz and Topulos [10]. In both cases the laser pulse saturates the optical transition and produces a small number of initial electrons. Then avalanche ionization develops without the presence of the laser ra- 
diation, using the energy stored in excited atoms. For such an excitation, the ionization of the vapor is only partial and does not exceed few percent. This model is improved by introducing the energy pooling and Penning ionization processes into the ionization scheme [11]. The spectroscopic observations of laser-produced plasmas in alkali-metal vapors induced by two-photon resonances using $10 \mathrm{~ns}$ pulsed laser have been reported [12]. More over, the majority of theoretical [13] and experimental studies on multi-photon ionization [14] of atoms was studied in atomic beams in the absence of any atomic collision processes.

Recently Bakhramov et al. [15], study the mutual competition of the processes which are responsible for the seeding electrons in the plasma produced by resonance excitation of rubidium vapor namely; two photon ionization, associative ionization and Penning ionization, using nanosecond pulses of laser radiation. Therefore, the present work aimed to investigate the actual contribution of each of these processes to the plasma generation in laser saturation of rubidium vapor. In doing so a numerical model is applied which includes photo ionization and collisional ionization of the saturated excited atoms, as electron seeding processes. In addition the model includes also electron impact and atom-atom collisional processes which leads eventually to plasma formation. The model is applied to investigate the experimental measurement that carried out by Bakhramov et al. [15] to study theoretically the mutual competition of the two photon-ionization, associated ionization and Penning ionization processes of rubidium atoms excited by nanosecond resonant laser pulses.

\section{Theoretical Approach}

Calculation are based on a previously developed $[16,17]$ numerical model which involves a system of rate equations that describe the variation of the population of the considered nineteen level $(5 d \leq n l \leq 11 s)$ of the rubidium atom in addition to atomic ion and molecular ion levels. In the present work the model is modified to take into account the pulsed nature of the laser beam. The model is applied to investigate the experimental conditions given by Bakhramov et al. [15]. In their experiment the rubidium vapor $\left(10^{11}-10^{14} \mathrm{~cm}^{-3}\right)$ was irradiated by resonance laser pulse of duration $\approx 30 \mathrm{~ns}$ (FWHM) and intensity varies between $10 \mathrm{~kW} \cdot \mathrm{cm}^{-2} \leq \mathrm{I} \leq 2 \mathrm{MW} \cdot \mathrm{cm}^{-2}$ with line width $\leq 0.25 \mathrm{~cm}^{-1}$ which is tuned to the $\mathrm{D}_{1,2}$-resonance line. The physical kinetic processes which are considered in our model and the values of the cross sections are indicated in Table 1.

\subsection{Saturation of the Atomic Transition}

The intensity of resonant laser radiation used for ionization $I(v)$ prove to be greater than the saturation intensity for the corresponding transition,

$$
I_{s}=h v / \sigma_{21}(v) \tau_{2}
$$

where $\sigma_{21}(v)$ is the absorption cross-section at the frequency $v$, and $\tau_{2}$ is the life time of the excited state. Under these conditions we can evaluate the characteristic time for establishing an equilibrium concentration of excited atoms:

$$
\tau_{2} \cong \frac{h v}{I \sigma_{21}}
$$

\subsection{Rate Equations}

The equations describing the temporal variation of the population density of Rb levels $5 s, 5 p$ and $n l$ were expressed in the form:

$$
\begin{aligned}
& \frac{\mathrm{d} N(5 s)}{\mathrm{d} t}= N(5 p)\left(R_{21}+A_{21}\right)-N(5 s) R_{12}+N(5 p) \int n_{e}(\varepsilon) K_{21}(\varepsilon) \mathrm{d} \varepsilon-N(5 s) \int n_{e}(\varepsilon) K_{12}(\varepsilon) \mathrm{d} \varepsilon+N(5 p) N(n) K_{P I} \\
&+\frac{1}{2} N^{2}(5 P) \sigma_{P L} \nu F+\frac{1}{2} N^{2}(5 p) K_{E P}-N(n) N(5 s) K_{H M I}-N(5 s) \int n_{e}(\varepsilon) K_{1 c}(\varepsilon) \mathrm{d} \varepsilon \\
& \frac{\mathrm{d} N(5 P)}{\mathrm{d} t}= N(5 s) R_{12}-N(5 p)\left(R_{21}+A_{21}\right)-N(5 p) \int n_{e}(\varepsilon) K_{21}(\varepsilon) \mathrm{d} \varepsilon+N(5 s) \int n_{e}(\varepsilon) K_{12}(\varepsilon) \mathrm{d} \varepsilon \\
&-\frac{1}{2} N^{2}(5 p) K_{A I}-N(5 p) N(n) K_{P I}-\frac{1}{2} N^{2}(5 p) \sigma_{P L} v F \\
&-\frac{1}{2} N^{2}(5 P) K_{E P}-N(5 p) \sigma_{2 c}^{(2)} F^{2}-N(5 s) \int n_{e}(\varepsilon) K_{2 c}(\varepsilon) \mathrm{d} \varepsilon \\
& \frac{\mathrm{d} N(n)}{\mathrm{d} t}=\sum_{n \succ m} n_{e}(\varepsilon) N(n) K_{n m}(\varepsilon)-\sum_{m \succ n} n_{e} N(n) K_{m n}(\varepsilon)-\sum_{n \succ m} A_{n m} N(n)-\sum_{n} n_{e}(\varepsilon) N(n) K_{n c}(\varepsilon)-\sum_{n} N(5 p) N(n) K_{P I} \\
&+ \frac{1}{2} N^{2}(5 p) K_{E P}-\sum_{n} N(n) N(5 s) K_{H M I}-\sum_{n \succ 2} N(n) \sigma_{n c}^{(1)} F+N_{R b^{+}} n_{e}(\varepsilon) \sum_{n}\left[n_{e}(\varepsilon) K_{c n}(\varepsilon)+K_{R D}(\varepsilon)\right]
\end{aligned}
$$


Table 1. Physical kinetic processes in laser excited rubidium atoms [21,22].

\begin{tabular}{|c|c|c|c|}
\hline Ionization process & $n l$ & Cross section & Ref \\
\hline \multirow{10}{*}{$\begin{array}{l}\text { Single photon ionization } \sigma^{(1)} \times 10^{-18}\left(\mathrm{~cm}^{2}\right) \\
R b(n l)+h v \rightarrow R b^{+}+e\end{array}$} & $6 p$ & 15 & 21 \\
\hline & $5 d$ & 16 & 21 \\
\hline & $7 p$ & 7.0 & 21 \\
\hline & $6 d$ & 8.0 & 21 \\
\hline & $8 s$ & 7.1 & 21 \\
\hline & $7 d$ & 5.7 & 21 \\
\hline & $9 s$ & 5.1 & 21 \\
\hline & $8 d$ & 3.9 & 22 \\
\hline & $10 s$ & 4.4 & 22 \\
\hline & $9 d$ & 2.7 & 22 \\
\hline \multirow{2}{*}{$\begin{array}{l}\text { Two photon ionization } \sigma^{(2)} 5 P_{J \rightarrow c} \times 10^{-47}\left(\mathrm{~cm}^{4} \cdot \mathrm{sec}\right) \\
R b(5 p)+2 h v \rightarrow R b^{+}+e\end{array}$} & $3 / 2$ & 2.8 & 15 \\
\hline & $1 / 2$ & 0.3 & 15 \\
\hline Associative ionization $\sigma_{A I} \times 10^{-16}\left(\mathrm{~cm}^{2}\right)$ & $3 / 2$ & 5.4 & 15 \\
\hline$R b(5 p)+R b(5 p) \rightarrow R b_{2}^{+}+e$ & $1 / 2$ & 0.14 & 15 \\
\hline \multirow{2}{*}{$\begin{array}{l}\text { Laser-assisted penning ionization } \sigma_{P L} \times 10^{-41} \quad\left(\mathrm{~cm}^{4} \cdot \mathrm{sec}\right) \\
R b(5 p)+R b(5 p)+h v \rightarrow R b(5 s)+R b^{+}+e\end{array}$} & $3 / 2$ & $4.5 \times 10^{2}$ & 15 \\
\hline & $1 / 2$ & 5.8 & 15 \\
\hline \multirow{3}{*}{$\begin{array}{l}\text { Hornbeck-Molnar ionization } \sigma_{H M I} \times 10^{-15}\left(\mathrm{~cm}^{2}\right) \\
R b(n l)+R b(5 s) \rightarrow R b_{2}^{+}+e\end{array}$} & $6 d$ & 3.4 & 6 \\
\hline & $8 s$ & 4.7 & 6 \\
\hline & $7 d$ & 4.9 & 6 \\
\hline \multirow{8}{*}{$\begin{array}{l}\text { Penning ionization } \sigma_{P I} \times 10^{-12}\left(\mathrm{~cm}^{2}\right) \\
R b(5 p)+R b(n l) \rightarrow R b(5 s)+R b^{+}+e\end{array}$} & $5 d$ & 3.5 & 6 \\
\hline & $6 d$ & 4.7 & 6 \\
\hline & $8 s$ & 1.6 & 6 \\
\hline & $7 d$ & 2.8 & 6 \\
\hline & $8 d$ & 3.1 & 6 \\
\hline & $10 s$ & 2.5 & 6 \\
\hline & $9 d$ & 2.6 & 6 \\
\hline & $11 s$ & 3.8 & 6 \\
\hline \multirow{2}{*}{$\begin{array}{l}\text { Energy pooling collisions } \sigma_{E P} \times 10^{-14}\left(\mathrm{~cm}^{2}\right) \\
R b(5 p)+R b(5 p) \rightarrow R b(5 s)+R b(5 d)\end{array}$} & $5 d$ & 3 & 5 \\
\hline & $5 d$ & 3 & 5 \\
\hline
\end{tabular}

where $\mathrm{R}_{21}\left(\mathrm{sec}^{-1}\right)$ represents the stimulated emission rate coefficient for transition from level 2 to 1 .

$$
R_{21}=B_{21} \int I(v) L_{21}(v) \mathrm{d} v / 4 \pi \cong(I(v) / h v) \sigma_{21}(v)
$$

$I(v)$ is the spectral irradiance of the radiation field at frequency $v$ appropriate to the $2 \rightarrow 1$ transition, $B_{21}$ represents Milne coefficient, and $L_{21}(v)$ represents the corresponding line profile function for the transition.

$A_{21}$ is the resonance transition Einstein coefficient for spontaneous emission. $N(5 s), N(5 p)$ and $N(n)$ represents the population density of $5 s, 5 p$ and $n l$ states respectively. While $n_{e}(\varepsilon)$ represents the free electron density as a function of electron energy $\varepsilon . K_{m n}\left(\mathrm{~cm}^{3} \cdot \mathrm{sec}^{-1}\right)$ represents the electron-collision rate coefficient for the $\mathrm{m} \rightarrow$ $n$ transition [18], $K_{n c}\left(\mathrm{~cm}^{3} \cdot \mathrm{sec}^{-1}\right)$ represents the electron collisional ionization rate coefficient for level $n$ [19], $K_{c n}$ $\left(\mathrm{cm}^{6} \cdot \mathrm{sec}^{-1}\right)$ represents the three body recombination rate coefficient [19], $K_{R D}\left(\mathrm{~cm}^{3} \cdot \mathrm{sec}^{-1}\right)$ represents the irradiative recombination rate coefficient to level $n$ [19].
$K_{A I}=\sigma_{A I} \nu$ and represent the rate coefficients of associative ionization;

$K_{H M I}=\sigma_{H M I} v$, represent the rate coefficient Hornbeck-Molnar ionization;

$K_{P I}=\sigma_{P I} \nu$ represents the rate coefficients Penning ionization;

$K_{E P}=\sigma_{E P} \nu$ represents the rate coefficient of energy pooling collisions.

Where $\sigma_{A I}, \sigma_{H M I}, \sigma_{P I}$ and $\sigma_{E P}$ are the cross sections of the associative ionization, Hornbeck-Molnar ionization, Penning ionization and energy pooling processes respectively.

$\sigma_{P L}$ is laser-assisted Penning ionization cross section, $v$ is the average velocity of atoms. $\sigma_{n c}^{(1)}$ is the singlephoton ionization cross section for level $n, \sigma_{2 c}^{(2)}$ is the two photon, resonance state ionization rate coefficient and $F$ represents the photon flux density.

The time evolution of the electron density as a function of electron energy $\varepsilon$ is given by, 


$$
\begin{aligned}
\frac{\mathrm{d} n_{e}(\varepsilon)}{\mathrm{d} t}= & \sum_{m \succ n} n_{e}(\varepsilon) N(m) K_{n m}(\varepsilon)-\sum_{m \prec n} n_{e}(\varepsilon) K_{m n}(\varepsilon)+\sum_{n} n_{e}(\varepsilon) N(n) K_{n c}(\varepsilon) \\
& +\sum_{n} N(5 p) N(n) K_{P I}+N(5 p) \sigma_{2 c}^{(2)} F^{2}+\sum_{n \supset 2} N(n) \sigma_{n c}^{(1)} F+\frac{1}{2} N^{2}(5 p) \sigma_{P L} v F \\
& +\frac{1}{2} N^{2}(5 p) K_{A I}+\sum_{n} N(n) N(5 s) K_{H M I}-N_{R b^{+}} n_{e}(\varepsilon) \sum_{n}\left[n_{e}(\varepsilon) K_{c n}(\varepsilon)+K_{R D}(\varepsilon)\right]
\end{aligned}
$$

The normalization conditions are given by

$$
\begin{aligned}
& N_{0}=\sum_{n} N(n)+N\left(R b^{+}\right) \\
& \int_{0}^{\infty} n_{e}(\varepsilon) \varepsilon^{1 / 2} \mathrm{~d} \varepsilon=1 \\
& \frac{\mathrm{d} N\left(R b^{+}\right)}{\mathrm{d} t}=\sum_{n} N(5 p) N(n) K_{P I}+\sum_{n} n_{e}(\varepsilon) N(n) K_{n c}(\varepsilon)+N(5 p) \sigma_{2 c}^{(2)} F^{2}+\sum_{n>2} N(n) \sigma_{n c}^{(1)} F \\
& +\frac{1}{2} N^{2}(5 p) \sigma_{P L} v F-N\left(R b^{+}\right) n_{e}(\varepsilon) \sum_{n}\left[n_{e}(\varepsilon) K_{c n}(\varepsilon)+K_{R D}(\varepsilon)\right] \\
& \frac{\mathrm{d} N\left(R b_{2}^{+}\right)}{\mathrm{d} t}=\frac{1}{2} N^{2}(5 p) K_{A I}+N(n) N(5 s) K_{H M I}
\end{aligned}
$$

where $N_{0}$ is the density of $\mathrm{Rb}$ atomic vapor.

In the above mentioned equations the factor $1 / 2$ appeared in the associative ionization, energy pooling and laser-assisted Penning ionization processes terms is considered to correct for possible double counting of each colliding pair of identical particles [20].

\section{Results and Discussion}

Calculations are carried out by solving numerically the set of rate Equations (1) to (11), for the exact resonance excitation of one-photon transitions of rubidium atoms between $5 \mathrm{~S}_{1 / 2} \rightarrow 5 \mathrm{P}_{3 / 2}$ or $5 \mathrm{~S}_{1 / 2} \rightarrow 5 \mathrm{P}_{1 / 2}$ under the experimental conditions of Bakhramov et al. [15]. Numerical simulations were performed for the different experimentally tested values of both initial rubidium atoms and laser radiation intensities, to study the mutual competition between the multi-photon ionization and the different collisional ionization processes and its contributions to the plasma generated during the interaction. The output of these calculations obtained the following:

- The ion density $\left(\mathrm{N}_{\mathrm{i}}\right)$ as function of laser intensity (I).

- The dependence of the ion density $\left(\mathrm{N}_{\mathrm{i}}\right)$ on rubidium atomic density $(\mathrm{Rb})$.

- Comparative study of the separate contribution of each of the following processes; associative ionization, photoionization (single and two) and laser induced Penning ionization, to the production and growth of the ion density $\left(\mathrm{N}_{\mathrm{i}}\right)$.

\subsection{Variation of the Ion Density with the Laser Intensity}

Figures 1-3 show relations of the ion density $\mathrm{N}_{\mathrm{i}}$ versus laser intensity for the resonant transition $5 \mathrm{~S}_{1 / 2} \rightarrow 5 \mathrm{P}_{3 / 2}$ at the three values of rubidium vapor density namely; $3 \times$ $10^{11} \mathrm{~cm}^{-3}, 3 \times 10^{12} \mathrm{~cm}^{-3}$ and $3 \times 10^{13} \mathrm{~cm}^{-3}$. From these figures it is clear that the behavior of the calculated ion density agrees with those experimentally measured by Bakhramov et al. [15]. For rubidium densities $3 \times 10^{11}$ $\mathrm{cm}^{-3}$ and $3 \times 10^{12} \mathrm{~cm}^{-3}$ (Figures 1 and 2) during the initial stage of ionization (at low laser intensity $<10^{6} \mathrm{~W} \cdot \mathrm{cm}^{-2}$ ), the results showed slopes 2 and 1.7 respectively. This indicates that in Figure 1 (the lower $\mathrm{Rb}$ density) the production of the seed electrons and formation of ion current proceeds mainly via two photon ionization process. While Figure 2 shows that associative ionization process in binary atom collisions also take part in the ion production. This result is consistent with the analysis of the $\mathrm{Rb}$ vapor under resonance irradiation given by Barbier and Cheret [6], where the later mechanism was used to explain the production of the photo-resonant plasma. In their experiment, the measured ion current is found to be proportional to the squared density of the $\mathrm{Rb}$ atoms. This indicates that associative ionization could play a significant role for the production of the initial charged particles at relatively low laser intensities. At the higher laser intensity region $>1.0 \mathrm{MW} \cdot \mathrm{cm}^{-2}$ a considerable growth of ion yield is achieved where the obtained slope over this region became 3. This could be explained on the bases that, as the laser intensity increases the density of the highly-excited atoms increase. This in turn increases the probability of another type of associative ionization process due to collision of atoms in these highly excited states with the resonantly-excited atoms in the $5 p$ state which is known as Hornbeck-Molnar 


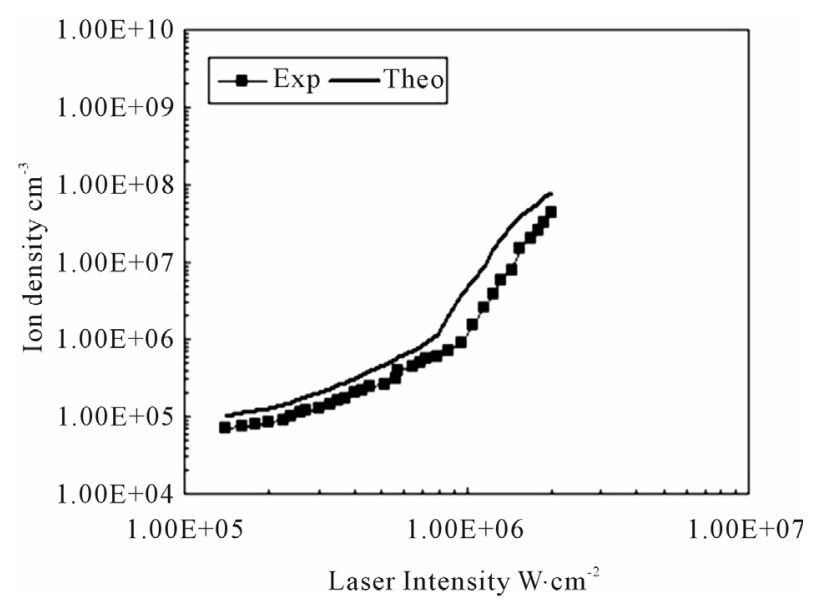

Figure 1. Variation of ion density of rubidium atoms with laser radiation intensity under excitation by nanosecond laser pulse to the resonant transition $5 S_{1 / 2}-5 P_{3 / 2}$ at $N_{R b}=3 \times 10^{11}$ $\mathbf{c m}^{-3}$.

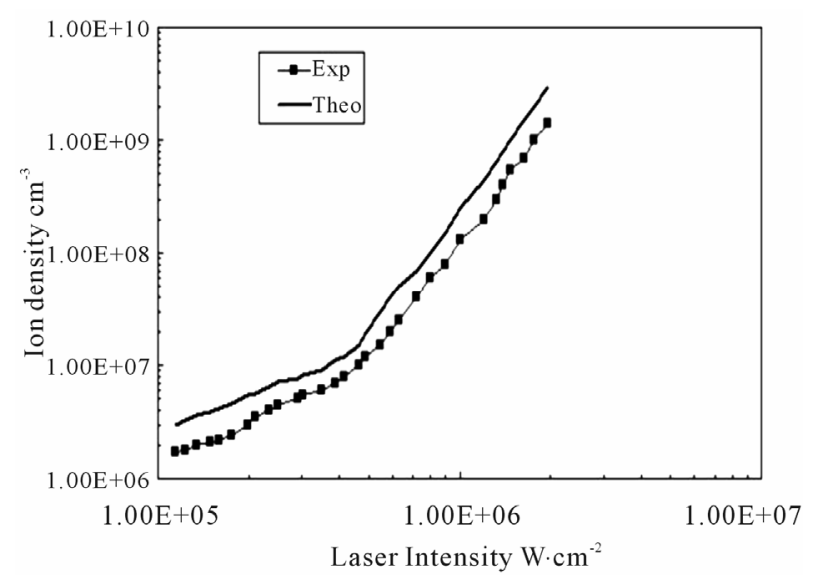

Figure 2. Variation of ion density of rubidium atoms with laser radiation intensity under excitation by nanosecond laser pulse to the resonant transition $5 S_{1 / 2}-5 P_{3 / 2}$ at different values of rubidium atom densities.

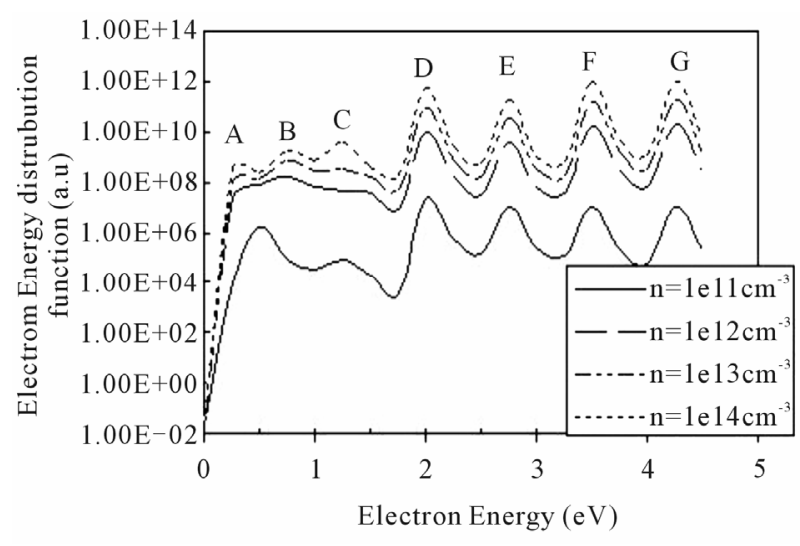

Figure 3. The distribution function of electrons in rubidium vapour irradiated by nanosecond laser pulse to the resonant radiation $5 S_{1 / 2}-5 P_{3 / 2}$ at different values of rubidium vapor densities. ionization [6,17]. In addition, these highly excited atoms can also contribute to the growth of the charged particles and hence ion current production through collision with the resonantly excited atoms in the $5 p$ state through Penning ionization process. Moreover, atoms in highly excited states can appear in collisions between two resonantly excited atoms by laser radiation, where the excitation energy of one atom can be partially transferred to the other-energy pooling collision. Therefore, rubidium atoms in highly excited states may be produced through this process, rather than step-by-step excitation of atoms by electrons impact Barbier and Cheret [5]. These processes which are included in the present calculations may explain the occurrence of the cubic dependence at higher laser intensities observed experimentally [15](Figures 1 and 2). The electrons which emerge due to the associative mechanism, rapidly gain energy in superelastic collisions with excited atoms add up the energy of laser photons $h v=1.58 \mathrm{eV}$ that results in the energy distribution with peaks separated by the energy of one photon as shown in Figure 3. The characteristic time of superelastic collisions for electrons of energy $E=0.25 \mathrm{eV}$ is between $4 \times 10^{-6} \mathrm{~s}$ and $4 \times 10^{-7} \mathrm{~s}$ for $N(5 p)=10^{12}-10^{13}$ $\mathrm{cm}^{-3}$. In general, the structure for the spectra shows that Penning ionization process is dominant over associative ionization process.

With the further increase of the rubidium density to a value of $3 \times 10^{13} \mathrm{~cm}^{-3}$ (Figure 4), the seed electrons are provided through the competition between laser induced ionization a result of two-photon ionization and paircollision of the resonantly excited rubidium atoms. In addition the process of laser-induced Penning ionization could be another possible mechanism for the production of atomic $\mathrm{Rb}^{+}$ions at the high rubidium density.

On the other hand, for the resonance transition $5 \mathrm{~S}_{1 / 2} \rightarrow$ $5 \mathrm{P}_{1 / 2}$ the relation between the ion density and laser intensity are plotted only for two values of the atomic vapor density; $1 \times 10^{13} \mathrm{~cm}^{-3}$ and $4 \times 10^{13} \mathrm{~cm}^{-3}$ and shown in Figures 5 and 6 respectively. For an easy comparison the experimentally measured values of Bakhramov et al. [15] are also shown on these figures. It is noticed that the behavior of the calculated ion density agrees reasonably with those measured ones over the examined range of the laser intensity. This again may be attributed to the various physical processes included in the model which depends on the population density of the highly excited states. In contrast to the behavior shown in Figures 1 and 2 for the case of $5 \mathrm{~S}_{1 / 2} \rightarrow 5 \mathrm{P}_{3 / 2}$ transitions, where the ion density starts growing slowly followed by a faster rate as the laser intensity increases, in Figures $\mathbf{5}$ and $\mathbf{6}$, the ion density increases linearly with the laser intensity. This linear behavior assures the dominant contribution of the process of laser-assisted Penning ionization in providing the initial electrons required for plasma generation. 


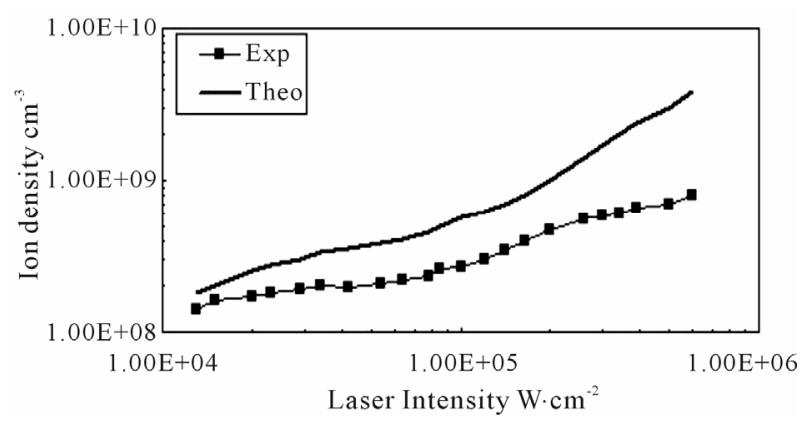

Figure 4. Variation of ion density of rubidium atoms with laser radiation intensity under excitation by nanosecond laser pulse to the resonant transition $5 \mathrm{~S}_{1 / 2}-5 \mathrm{P}_{3 / 2}$ at $\mathrm{N}_{\mathrm{Rb}}=3 \times$ $10^{13} \mathrm{~cm}^{-3}$.

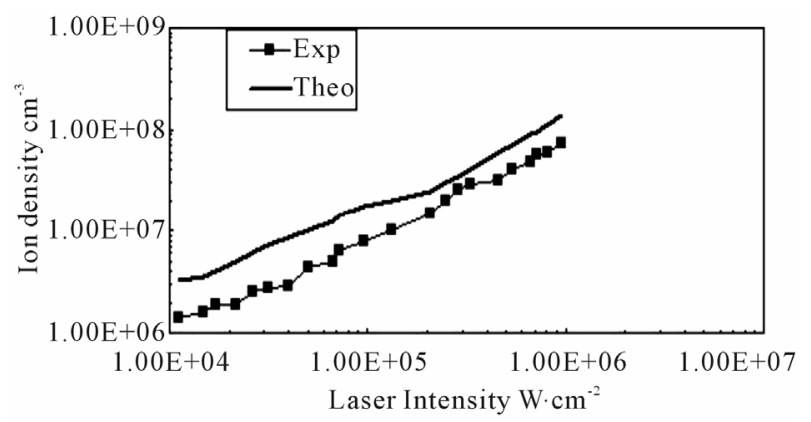

Figure 5. Variation of ion density of rubidium atoms with laser radiation intensity under excitation by nanosecond laser pulse to the resonant transition $5 S_{1 / 2}-5 P_{1 / 2}$ at $N_{R b}=1 \times$ $10^{13} \mathrm{~cm}^{-3}$.

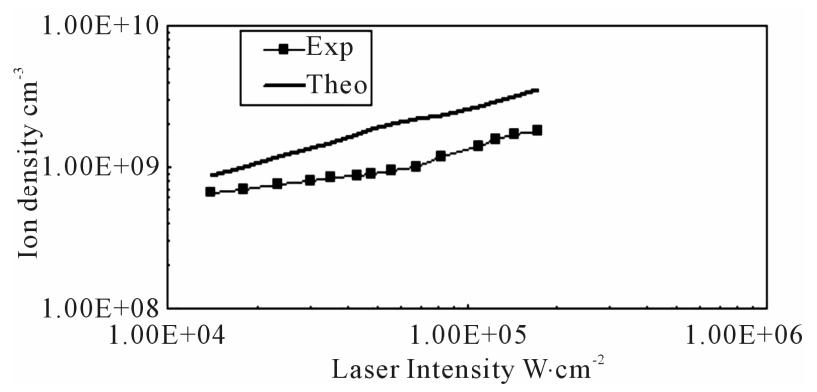

Figure 6. Variation of ion density of rubidium atoms with laser radiation intensity under excitation by nanosecond laser pulse to the resonant transition $5 S_{1 / 2}-5 P_{1 / 2}$ at $N_{R b}=4 \times$ $10^{13} \mathrm{~cm}^{-3}$.

No evidence is obtained in this figure for the contribution of the two photon ionization process to the production of the ion density. This result is confirmed from the noticeable increase of the ion density with the increase of the rubidium atomic density over the whole range of the laser intensity as shown in Figure 5.

\subsection{Ion Density Dependence on the Rubidium Atomic Density}

Comparison between the experimental and theoretical values of the ion density $\mathrm{N}_{\mathrm{i}}$ as a function of rubidium atomic density for the transition $5 \mathrm{~S}_{1 / 2} \rightarrow 5 \mathrm{P}_{3 / 2}$, at two values of the laser intensity $35 \mathrm{~kW} \cdot \mathrm{cm}^{-2}$ and 500 $\mathrm{kW} \cdot \mathrm{cm}^{-2}$ is presented in Figures 7 and $\mathbf{8}$ respectively. It can be seen from these figures that, the trend of the calculated values agree well with the experimentally measured ones of Bakhramov et al. [15] both show linear behavior of the ion density versus the rubidium atomic density. This agreement clarified the main contribution of the two photon ionization and associative ionization to the ion density yield. This confirms the quadratic dependence of the ion density $\mathrm{N}_{\mathrm{i}}(\mathrm{I})$ on the laser intensity shown in Figures 1 and 2 . In contrast, for the $5 \mathrm{~S}_{1 / 2} \rightarrow$ $5 \mathrm{P}_{1 / 2}$ transition, the ion yield dependence is found to be essentially nonlinear as shown in Figure 9. This indicates that collisional processes such as associative ionization and laser assisted Penning ionization are two of the main processes which contribute to the production of the ion density in laser excited rubidium vapor.

\subsection{Comparative Study of the Ionization Processes}

For analyzing the ionization of the alkali metal vapor under the influence of resonant laser radiation, it is of great interest to reveal the separate contribution of each of the following processes; associative ionization, photoionization (single and two) and laser induced Penning ionization, to the production and growth of the ion density. Figures $\mathbf{1 0}$ and $\mathbf{1 1}$ represent comparison between the ion density produced by each of these processes as a function rubidium atomic density at the two considered values of the laser intensity namely; $35 \mathrm{~kW} \cdot \mathrm{cm}^{-2}$ and 500 $\mathrm{kW} \cdot \mathrm{cm}^{-2}$ respectively. From Figure 10, it is noticed that, at the low laser power, associative ionization is the major process responsible for the production of the ion density.

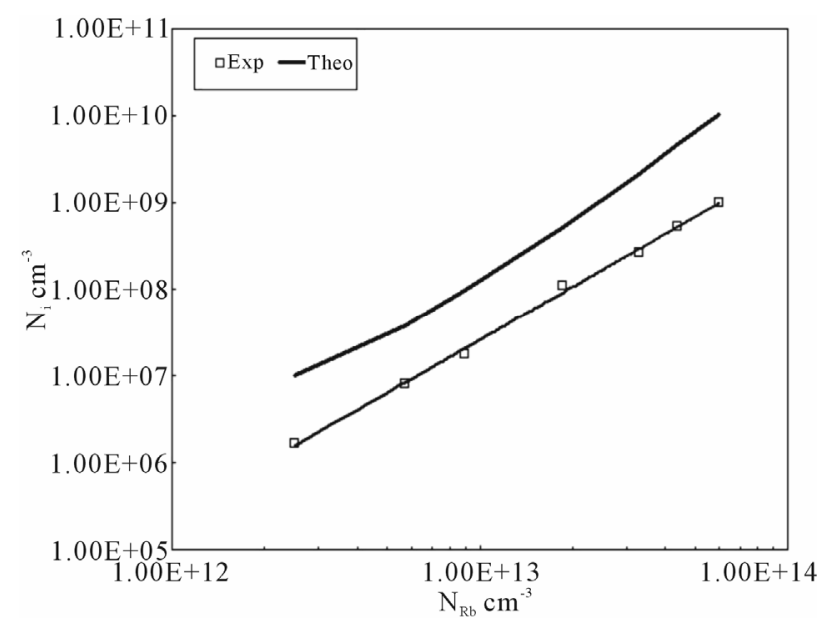

Figure 7. The ion density of versus rubidium atom density for the resonant transition $5 S_{1 / 2}-5 P_{3 / 2}$ at laser intensity $I=$ $35 \mathrm{~kW} \cdot \mathrm{cm}^{-2}$. 


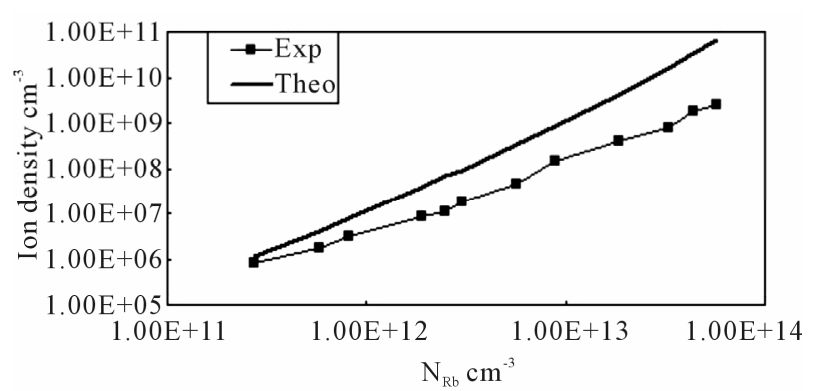

Figure 8. The ion density of versus rubidium atom density for the resonant transition $5 S_{1 / 2}-5 P_{3 / 2}$ at laser intensity $I=$ $500 \mathrm{~kW} \cdot \mathrm{cm}^{-2}$.

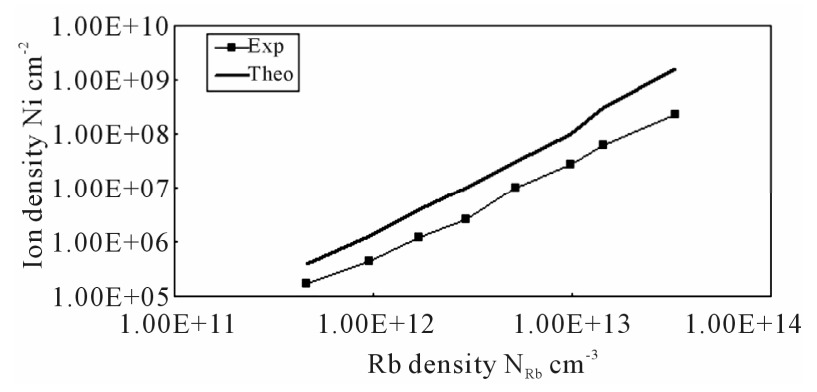

Figure 9. The ion density of versus rubidium atom density for the resonant transition $5 S_{1 / 2}-5 P_{1 / 2}$ at laser intensity $I=$ $500 \mathrm{~kW} \cdot \mathrm{cm}^{-2}$.

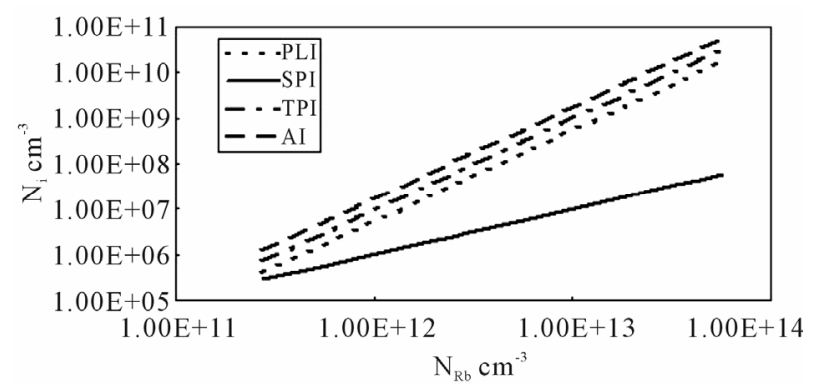

Figure 10. Comparison between the ion density versus rubidium atom density for the separate contribution of associative ionization (AI), two photon-ionization (TPI), laser assisted Penning Ionization (PLI) and single photon ionization (SPI) processes for the resonant transition $5 \mathrm{~S}_{1 / 2}-5 \mathrm{P}_{3 / 2}$ at laser intensity $I=35 \mathrm{~kW} \cdot \mathrm{cm}^{-2}$.

This means that molecular ions are dominant over the atomic ones. The density of the molecular ions is proportional to the squared density of rubidium atomic density. This confirms the experimental observation of Bakhramov et al. [15]. Two photon-ionization of the resonantly excited atoms and laser assisted Penning ionization processes may also play significant role in the production of the atomic ions $[6,15]$. Single photon-ionization is found to have the least contribution.

At the higher laser intensity, $500 \mathrm{~kW} \cdot \mathrm{cm}^{-2}$, Figure 11 shows that the ion yield may be provided by laser assisted Penning ionization and two photon ionization

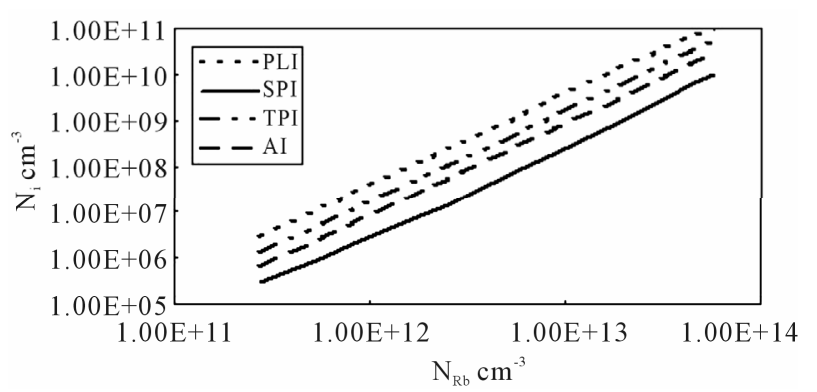

Figure 11. Comparison between the ion density versus rubidium atom density for the separate contribution of associative ionization (AI), two photon-ionization (TPI), laser assisted Penning Ionization (PLI) and single photon ionization (SPI) processes for the resonant transition $5 S_{1 / 2}-5 P_{3 / 2}$ at laser intensity $\mathrm{I}=500 \mathrm{~kW} \cdot \mathrm{cm}^{-2}$.

processes. The rate of growth of these processes increases as the atomic vapor density increases. Associative ionization contributes also to the molecular ions production. Contrary to the case of low laser intensity, single photon ionization process showed a noticeable contribution to the atomic ions growth rate.

\section{Conclusion}

The problem of laser-induced resonance of rubidium vapor tuned to the resonance lines $D_{1}$ or $D_{2}$ is investigated. A numerical model is applied which includes the Boltzmann equation for the electron energy distribution function, as well as a system of rate equations describing the variation of the excited states population that formed during the interaction of a resonantly tuned laser beam with $\mathrm{Rb}$ vapor in addition to the ions current rate of growth. The results presented in this study are summarized as follows: 1) The validity of the more realistic approach given by the model to interpret the findings of laser induced resonance excitation of alkali metal vapors experiments with pulsed laser sources that carried by Bakhramov et al. [15]; 2) For both transitions $D_{1,2}$ at lower vapor densities $\left(3 \times 10^{11} \mathrm{~cm}^{-3}\right.$ and $\left.3 \times 10^{12} \mathrm{~cm}^{-3}\right)$ the ionization of the vapor proceeds via two photon ionization process together with associative ionization process which is found to be also effective. While at the higher vapor density ionization by collisional processes is dominant; 3) The investigation of ion yield dependence on $\mathrm{Rb}$ density at two fixed values of the laser intensity, for the transition $5 \mathrm{~S}_{1 / 2} \rightarrow 5 \mathrm{P}_{3 / 2}$ revealed that both quadratic ion yield dependence at the low laser intensity and linear behavior versus $\mathrm{Rb}$ density confirms the assumption of two photon ionization channel to be favorable at low vapor density. In contrast for the $5 \mathrm{~S}_{1 / 2} \rightarrow$ $5 \mathrm{P}_{1 / 2}$ transition, the ion yield dependence is essentially nonlinear and indicates that collisional ionization processes give the major contribution to the total ionization; 4) Finally, comparison between the ion yield produced for 
the separate contribution of each of the ionization processes at the two fixed laser intensities showed that at low intensity, associative ionization is found to be the main channel for the ion production, this process provides molecular ions. At the high laser intensity the ion current produced through two photon ionization process at $\mathrm{Rb}$ densities $<10^{12} \mathrm{~cm}^{-3}$, laser assisted collisional ionization processes are responsible for the ion yield at higher $\mathrm{Rb}$ densities.

\section{REFERENCES}

[1] A. N. Klyucharev, "Chemi-Ionization Processes," Physics-Uspekhi, Vol. 36, No. 6, 1993, pp. 486-512.

[2] T. B. Lucatorto and T. J. McIlrath, "Laser Excitation and Ionization of Dense Atomic Vapors," Applied Optics, Vol. 19, No. 23, 1980, pp. 3948-3956. doi:10.1364/AO.19.003948

[3] A. N. Klucharev, N. N. Bezuglov, A. A. Matveev, A. A. Mihajlov, Lj. M. Ignjatovic and M. S. Dimitrijevic, "Rate Coefficients for the Chemi-Ionization Processes in Sodium- and other Alkali-Metal Geocosmical Plasmas," New Astronomy Reviews ,Vol. 51, No. 7, 2007, pp. 547-562.

[4] M. Cheret, A. Spielfiedel, R. Durand and R. Deloche, "Collisional Ionisation of Highly Excited Rubidium S State," Journal of Physics B: Atomic and Molecular Physics, Vol. 14, No. 20, 1981, pp. 3953-3959. doi:10.1088/0022-3700/14/20/019

[5] L. Barbier and M. Cheret, "Energy Pooling Process in Rubidium Vapour," Journal of Physics B: Atomic and Molecular Physics, Vol. 16, No. 17, 1983, pp. 3213-3228. doi:10.1088/0022-3700/16/17/014

[6] L. Barbier and M. Cheret, "Experimental Study of Penning and Hornbeck-Molnar Ionization of Rubidium Atoms Excited in a High s or d Level $(5 d \leq n l \leq 11 s)$," Journal of Physics B: Atomic and Molecular Physics, Vol. 20, No. 6, 1987, pp. 1229-1248. doi:10.1088/0022-3700/20/6/011

[7] M. Cheret and L. Barbier, "Collisional Ionization of Excited Rubidium Atoms," Journal De Physique, Vol. 46, No. 1, 1985, pp. 193-197.

[8] R. M. Measures, P. L. Wizinowich and P. G. Cardinal, "Fast and Efficient Plasma Heating through Superelastic Laser Energy Conversion," Journal of Applied Physics, Vol. 51, No. 7, 1980, pp. 3622-3628. doi: $10.1063 / 1.328142$

[9] J. L. Bobin and M. A. Zaibi, "Inversions de Population lors de la Création, par Interaction Laser Résonnante, de Plasmas Complètement Ionisés," Journal de Physique Lettres, Vol. 46, No. 16, 1985, pp. 737-744. doi:10.1051/jphyslet:019850046016073700.

[10] T. Stacewicz and W. Latek, "Ionization of Sodium Vapour by Nanosecond Resonant Laser Pulses Tuned to 3s to 4p Transition," Physica Scripta, Vol. 42, No. 6, 1990, pp. 658-660.

[11] P. Bicchi, "Energy Pooling Reactions," La Rivista del Nuovo Cimento, Vol. 20, No. 7, 1997, pp. 1-74. doi:10.1007/BF02879250

[12] W. C. Stwalley and J. T. Bhans, "Atomic, Molecular, and Photonic Processes in Laser-Induced Plasmas in Alkali Metal Vapors," Laser and Particle Beams, Vol. 11, No. 1, 1993, pp. 185-204.

[13] E. W. McDaniel, “Atomic Collisions," Wiley, New York, 1998.

[14] T. J. Moseley, "Photodissociation and Photoionization Processes," Wiley, New York, 1994.

[15] S. A. Bakhramov, E. V. Vaganov, A. M. Kokhkharov and O. V. Parpiev, "Laser-Induced Resonant Multiphoton and Collisional of Rubidium Atoms," Proceeding of SPIE, Vol. 4748, 2002, pp. 205-210. doi:10.1117/12.468948

[16] M. A. Mahmoud, "Electron Energy Distribution Function in Laser-Excited Rubidium Atoms," Journal of Physics B: Atomic, Molecular \& Optical physics, Vol. 38, No. 10, 2005, pp. 1545-1556.

[17] M. A. Mahmoud, "Kinetics of $\mathrm{Rb}_{2}^{+}$and $\mathrm{Rb}^{+}$Formation in Laser-Excited Rubidium Vapor," Central European Journal of Physics, Vol. 6, No. 3, 2008, pp. 530-538. doi:10.2478/s11534-008-0074-5

[18] H. W. Drawin, "Rapport Euratom CEA.92 Fontenay-AuxRoses (France) FC," 1967.

[19] J. L. Vriens, "Energy Balance in Low-Pressure Gas Discharges," Journal of Applied Physics, Vol. 44, No. 9, 1973, pp. 3980-3989.

[20] N. N. Bezuglov, A. N. Klyucharev and V. A. Sheverev, "Associative Ionization Rate Constants Measured in Cell and Beam Experiments," Journal of Physics B: Atomic and Molecular Physics, Vol. 20, No. 11, 1987, pp. 24972513. doi:10.1088/0022-3700/20/11/018

[21] M. Aymar, E. Luc-Koenig and F. Combet-Farnoux, "Theoretical Investigation on Photoionization from Rydberg States of Lithium, Sodium and Potassium," Journal of Physics B: Atomic and Molecular Physics, Vol. 9, No. 8, 1976, pp. 1279-1291.

[22] M. Aymar, O. Robaux and S.Wane, "Central-Field Calculations of Photoionisation Cross Sections of Excited States of $\mathrm{Rb}$ and $\mathrm{Sr}^{+}$and Analysis of Photoionisation Cross Sections of Excited Alkali Atoms Using Quantum Defect Theory," Journal of Physics B: Atomic and Molecular Physics, Vol. 17, No. 6, 1984, pp. 993-1007. 